\title{
Disappearance of the inversion effect during memory-guided tracking of scrambled biological motion
}

\author{
Changhao Jiang ${ }^{1} \cdot$ Guang H. Yue ${ }^{2} \cdot$ Tingting Chen $^{3} \cdot$ Jinhong Ding ${ }^{3}$
}

Published online: 29 February 2016

(C) Psychonomic Society, Inc. 2016

\begin{abstract}
The human visual system is highly sensitive to biological motion. Even when a point-light walker is temporarily occluded from view by other objects, our eyes are still able to maintain tracking continuity. To investigate how the visual system establishes a correspondence between the biological-motion stimuli visible before and after the disruption, we used the occlusion paradigm with biological-motion stimuli that were intact or scrambled. The results showed that during visually guided tracking, both the observers' predicted times and predictive smooth pursuit were more accurate for upright biological motion (intact and scrambled) than for inverted biological motion. During memory-guided tracking, however, the processing advantage for upright as compared with inverted biological motion was not found in the scrambled condition, but in the intact condition only. This suggests that spatial location information alone is not sufficient to build and maintain the representational continuity of the biological motion across the occlusion, and that the object identity may act as an important information source in visual tracking. The inversion effect disappeared when the scrambled biological motion was occluded, which indicates that when biological motion is temporarily occluded and there is a
\end{abstract}

Jinhong Ding

dingih@263.net

Changhao Jiang

jiang20141219@163.com

1 Beijing Key Laboratory of Physical Fitness Evaluation and Technical Analysis, Capital University of Physical Education and Sports, Beijing, China

2 Human Performance and Engineering Research, Kessler Foundation, West Orange, NJ, USA

3 Department of Psychology, Capital Normal University, Beijing, China complete absence of visual feedback signals, an oculomotor prediction is executed to maintain the tracking continuity, which is established not only by updating the target's spatial location, but also by the retrieval of identity information stored in long-term memory.

Keywords Biological motion - Inversion effect · Predictive smooth pursuit $\cdot$ Occlusion paradigm $\cdot$ Identity-location bindings

Our eyes have an amazing ability to accurately predict the velocity and future position of visual targets in motion, such as a running man. Even when a moving target is temporarily occluded from view by other objects and then reappears-for instance, a man reappears after walking behind a car-we are able to maintain eyetracking continuity. Two kinds of eye movements make this ability possible: predictive smooth pursuit and catch-up saccades. For a target moving with a predictable trajectory (e.g., constant velocity or sinusoidal motion), smooth pursuit with gain close to unity can rapidly be achieved (Leigh \& Zee, 2006; Lisberger \& Fuchs 1978). However, in our natural surroundings, a target's trajectory is often unpredictable (e.g., accelerated motion or changing direction), so that eye velocity decreases and position error accumulates. To correct for this, catch-up saccades are often generated to compensate for the variability of the smooth-pursuit response, by adjusting the amplitude of saccades to the gain in the smooth-pursuit response (Orban de Xivry, Bennett, Lefèvre, \& Barnes, 2006). Therefore, the smooth-pursuit system needs to collaborate with the saccadic system in order to improve the tracking continuity of a target that moves in an unpredictable way.

Oculomotor prediction can be observed from the transient occlusion of simple object motion. For example, during the transient disappearance of a red laser spot moving along a 
circular path (Orban de Xivry, Missal, \& Lefèvre, 2008) or a white dot following a 2-D sinusoidal pattern (Barnes, Barnes, \& Chakraborti, 2000; Ding, Powell, \& Jiang, 2009), decay in the smooth eye velocities at the beginning of target disappearance was often accompanied by the release of one or more predictive saccades. However, the eye velocity predictably increased before target reappearance (Bennett \& Barnes, 2003; Coppe, Orban de Xivry, Missal, \& Lefèvre, 2010; Orban de Xivry et al., 2006). Alterations in target shape or color during occlusion did not significantly increase the frequency of disruptions in tracking (Muller \& Aslin, 1978). However, the predictive mechanism in ocular pursuit during transient occlusion was influenced by such low-level factors as pre- and postocclusion target velocity (Becker \& Fuchs, 1985; Mrotek \& Soechting, 2007; Orban de Xivry et al., 2006) and preocclusion viewing time (Bennett, Orban de Xivry, Barnes, \& Lefèvre, 2007; Orban de Xivry, Missal, \& Lefèvre, 2009). According to Barnes and Collins (2008), during sustained pursuit of a continuously visible target, retinal and extraretinal signals work together to maintain a stable response with high gain. Retinal input comes from direct feedback of visual motion signals and has an inherent visuomotor delay of 80-100 ms (Carl \& Gellman, 1987). Extraretinal inputs may reflect several real or hypothetical influences, including efference copy ("eye velocity memory"), remembered target motion ("target velocity memory"), volition, attention, and expectation (Bennett \& Barnes, 2004). However, when a target disappears very briefly behind an occluder, pursuit is maintained by the efference copy loop, acting as a memory of the eye velocity immediately prior to target disappearance (Churchland, Chou, \& Lisberger, 2003). It has been recognized that the extraretinal input is influenced by cognitive factors such as perception, expectation, and attention (Beutter \& Stone, 1998; Madelain \& Krauzlis, 2003; Pola \& Wyatt, 1997; Tanaka \& Lisberger, 2001).

The oculomotor system also exhibits reasonable tracking continuity during the temporary occlusion of some biologically meaningful objects, such as the human face. During a multiple-face tracking task, faces were immediately occluded by some identical objects after randomly moving for several seconds. The participants were not required to learn the identity of the faces; instead, they were required only to perform the tracking task (Ren, Chen, Liu, \& Fu, 2009). In a behavioral experiment, participants were able to track two human faces at most (Ren et al., 2009), which was similar to the results of tracking other biologically meaningful objects, such as cartoon animals (Horowitz et al., 2007). Furthermore, Ren et al. compared tracking performance both for different faces and for identical faces, and also included a condition in which the different faces were presented upside down. As they predicted, when the faces were immediately masked by a blank screen after randomly moving for $7 \mathrm{~s}$, participants were more accurate to identify the inverted different faces than the upright different faces (Ren et al., 2009). Ren et al. proposed that upright faces engage attentional resources for identity processing to some extent, which in turn impairs tracking performance. This impairment for upright faces may be caused by voluntary or mandatory face identity processing. This experimental finding suggests that during transient occlusion and in the absence of visual feedback signals, the identity of upright human faces may be processed even when identity processing is task-irrelevant. This is consistent with the findings of Horowitz et al. in an object-tracking task and provides strong evidence for the "object file" theory (Kahneman, Treisman, \& Gibbs, 1992), which assumes that identity encoding takes place within a capacity limit and allows for content-addressable information. The object file concept as a temporary episodic representation was developed to explain the perceptual continuity of objects over time. The maintenance of the perceived continuity of objects as they move, change, or momentarily disappear requires operations that link and integrate the current state of the object to its prior history. When an object is first attended, an object file is opened for it, and all subsequent information about that object goes into the object file. The object file is capable of restoring continuity that has been briefly broken in the stream of sensory inputs.

The object file theory proposed by Kahneman, Treisman, and Gibbs (1992) implies a two-process account of tracking, which was clarified by Oksama and Hyönä (2008) in details. They described a process that involves the serial, nonautomatic, and effortful updating of identity-location bindings (i.e., what-where bindings). Location information comes from spatial indices, temporarily stored in visual short-term memory (VSTM), and identity is from long-term memory (LTM) representations (Oksama \& Hyönä, 2008). Location information is always out of date and must be updated because of the spatial coordinate displacement between actual target locations and the target location information stored in VSTM. However, the target identity is precisely what the target retains, even as its properties and the label or name we give it vary. Identity is a collection of features and could be differentiated by shape and color (Bremner, Slater, Mason, Spring, \& Johnson, 2013), semantic category (Horowitz et al., 2007; Yu, 2000), and global configuration (Botterill, Allen, \& McGeorge, 2010; De Caro, 1998; Ren et al., 2009). Object identity is the property by which each object (regardless of its class or current state) can be identified and treated as a distinct entity. There is "something unique" about any object that distinguishes it from other objects (Khoshafian \& Copeland, 1986). Identity-location bindings are presumed to be held in working memory's episodic buffer and help to maintain the perceived continuity of objects as they move, change, or momentarily disappear.

When upright and inverted human body movements are used in an occlusion paradigm, however, the results are more 
complicated. Visual perception of human body movements has traditionally been investigated with a type of biologicalmotion stimulus, the point-light walker, composed of a number of point-lights depicting the motions of an agent's major joints (Johansson, 1973). Like the human face, the point-light walker is a biologically meaningful visual pattern, from which observers can readily extract action (Dittrich, 1993; Norman, Payton, Long, \& Hawkes, 2004), gender (Kozlowski \& Cutting, 1977; Mather \& Murdoch, 1994; Troje, 2002), and identity (Hill \& Pollick, 2000; Troje, Westhoff, \& Lavrov, $2005)$ information. When the point-light walker transiently disappears behind an occlusion and there is a complete absence of visual feedback signals, participants' eye velocity decays rapidly, but often it gradually recovers to the previous level before the end of occlusion (Coppe et al., 2010). Moreover, for every $50 \mathrm{~ms}$ throughout the occlusion, the smooth-pursuit velocity of human observers does not reveal any significant difference between upright and inverted pointlight walkers (Coppe et al., 2010). The absence of a difference for upright versus inverted point-light walkers in velocity gains during memory-guided pursuit, combined with the stronger visually guided smooth-pursuit reacceleration after reappearance of the upright in comparison with the inverted point-light walker, suggests that during transient occlusion of biological motion, oculomotor prediction is primarily driven by extraretinal inputs that contain a representation of the target spatial location (Bennett, Orban de Xivry, Lefèvre, \& Barnes, 2010; Orban de Xivry et al., 2008), independently from the identity representation stored in LTM (Bennett et al., 2010).

Facial stimuli and biological-motion stimuli are special visual patterns with obvious biological significance. The presence of a pronounced inversion effect in biological-motion perception (Barclay, Cutting, \& Kozlowski, 1978; Bertenthal \& Pinto, 1994; Dittrich, 1993; Shipley, 2003) is similar to the inversion effect found for identifying human faces (see Farah, Tanaka, \& Drain, 1995, for a review). The literature seems to suggest that the recovery of identity information, which is often defined by global configuration (Botterill, Allen, \& McGeorge, 2010; De Caro, 1998; Ren et al., 2009), plays a key role in biologically significant information processing (Bertenthal \& Pinto, 1994; Pavlova \& Sokolov, 2000; Sumi, 1984; Troje, 2002). However, these findings from tracking studies using biological-motion stimuli seem to be inconsistent with those from tracking studies using facial stimuli. In a study by Coppe et al. (2010), when a point-light walker (upright or inverted) transiently disappeared behind an occlusion, predictive smooth ocular pursuit decayed rapidly due to the complete absence of visual feedback signals, and extraretinal input continued to drive smooth pursuit at a reduced gain. Attention to the upright point-light walker causes a set of nodes in LTM that represent target identity to be activated, and all history information about that target goes into the object file, which is capable of restoring continuity that has briefly been broken in the stream of sensory inputs. In contrast to the upright point-light walker, the inverted point-light walker appeared for $800 \mathrm{~ms}$, which was not enough to ensure sufficient processing time for participants to build the identity representation and the identity-location binding of that global novelty target (the inverted point-light walker; Kahneman et al., 1992). However, the inverted human figures, as novelty stimuli, to some extent engaged more attentional resources, which in turn maintained tracking continuity during the transient absence of sensory inputs. In other words, the processing advantages of biological meaningfulness or significance (arising from upright biological-motion signals) during memory-guided pursuit might have been offset by the attentional effects of a novelty stimulus (arising from the inversion of human figures).

In this study, we sought to repeat Coppe et al.'s (2010) paradigm with a number of important changes. First and foremost, we used both intact and scrambled biological motion, which were randomly presented in upright and inverted patterns. Second, given that a higher reduction in eye velocity during the blanking phase would require greater involvement of the identity-location binding during the visible phase, we extended the exposure time of the stimuli. Specifically, the intact or scrambled biological-motion stimuli were visible for $4 \mathrm{~s}$ and were blanked temporarily for another $4 \mathrm{~s}$. Finally, to avoid the ceiling effect of observers predicting when the point-light walkers would come out from the occluder, the stimuli did not reappear after the transient occlusion. We hypothesized that if identity information were processed during memory-guided tracking, a significant inversion effect would occur in the intact biological-motion condition. In the scrambled biological-motion condition, since identity representations could not be established by an upright point-light walker or its inverted version, there would be no inversion effect.

\section{Method}

\section{Participants}

Twenty right-handed young undergraduate or graduate students (12 females, eight males; $20-30$ years of age) participated in this experiment. They reported normal or corrected-tonormal visual acuity and had no known cognitive or neurological disorders. All participants provided informed consent for procedures approved by the Academic Affairs Committee, Department of Psychology at Capital Normal University, and were paid for their participation.

\section{Design and stimuli}

A $2 \times 2 \times 2$ within-subjects factorial design was used for the experiment, with the first factor referring to the type of tracking point-light walker (intact or scrambled), the second to the 
target orientation (upright or inverted), and the third to the target visibility (visible or occluded). The intact point-light walkers were generated and presented using MATLAB (MathWorks, Natick, MA) by implementing Cutting's (1978) classic algorithm. A set of 12 light markers was used, which represented the head, one shoulder, one hip, one wrist, two elbows, two hands, two knees, and two feet (Fig. 1). This algorithm created a point-light display of a human walking in sagittal view, which animated each of the 12 dots with some combination of ellipsoid and pendular motion, defined within the hierarchical organization of an articulated body. The trajectories of shoulders and hips were modeled as simple ellipses, whereas the motion of the elbow and knee was modeled as that of two pendulums, with the motion of the shoulders and hips added to it (Cutting, 1978; Saunders, Suchan, \& Troje, 2009). Scrambled point-light walkers were created by randomizing the starting position of each point within the region approximately covered by the intact biologicalmotion sequence. Inverted point-light walkers (i.e., the intact and scrambled versions) were created by mirror-flipping all of the motion sequences vertically.

Stimuli were presented in white on a homogeneous gray background with a dark gray occlusion bar (Fig. 2). Across all trials, the point-light walker (i.e., the intact or scrambled versions), randomly presented in upright or inverted orientation, regularly moved from left to right across the screen on the gray region until it disappeared behind the dark gray occlusion bar. The visual display (visible phase plus occluded bar) subtended $25.99^{\circ}$ (horizontally) $\times 19.64^{\circ}$ (vertically) of visual angle, which was on for $10 \mathrm{~s}$ for each trial. The point-light walker subtended approximately $2.43^{\circ} \times 3.81^{\circ}$ of visual angle. Each individual light marker was $0.20^{\circ}$ in diameter and moved with the velocity of $15 \%$ s from the current location to the next location within a specific circle, following Cutting's (1978) computational program.

\section{Behavioral procedures}

The total 176 experiment trials were divided into four blocks, with each block having 44 trials. The order of trials within each block was randomized. Each trial lasted for 10,000 ms. Blocks were separated by 2-min breaks. At the beginning of the experiment, the instructions were presented and clarified to the participants.

Each trial began with a white "+" measuring $0.2^{\circ}$ of visual angle appeared on the left side of the screen as a cue and would be replaced by a point-light walker after $1,000 \mathrm{~ms}$. The observers were asked to focus on this "+" and then to follow the point-light walker with their eyes, tracking its motion for $8,000 \mathrm{~ms}$. The point-light walker always moved from left to right. The visible phase and the occlusion bar were presented for 4,000 ms, respectively. Participants were told that behind the occluded bar, the point-light walker continued to move with the same velocity. They were asked to press the left-hand button to indicate when the first light marker entered the left side of the dark gray occlusion bar and to press the button again when they predicted that the same light marker should reappear from the right side of the occlusion bar. For each experimental condition, the first light marker to enter the occlusion bar was identical to the one that should reappear from the right side of the occlusion bar. After the occlusion period, the moving target did not reappear until a $1,000-\mathrm{ms}$ waiting period was over. Participants were instructed to press the button as quickly as possible while minimizing errors. Before the formal experiment, the participants had completed 16 practice trials until they were familiar with the task and the stimuli.

\section{Oculomotor recording and analysis}

The participants were seated at a viewing distance of $60 \mathrm{~cm}$ from a 17-in. CRT computer screen. Their eye positions were measured with the SMI iView X RED Remote Eye-Tracking Device. The iView X RED-250 is a remote tracking system that calculates gaze using the reflection of a near-infrared light from the cornea and pupil of one eye with a sampling rate of $250 \mathrm{~Hz}$. Nine-point calibrations were performed at the beginning of each block. The eyetracking computer was synchronized to the MATLAB computer via a parallel port cable.
Intact
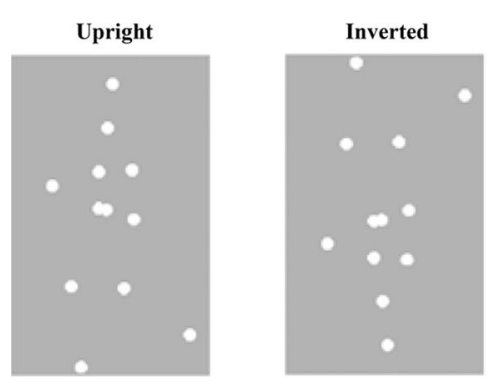

Scrambled

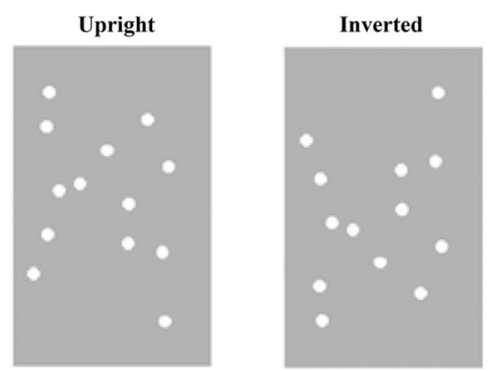

Fig. 1 Four types of point-light biological-motion stimuli: intact upright and inverted biological-motion stimuli (left panel) and scrambled upright and inverted biological-motion stimuli (right panel) 


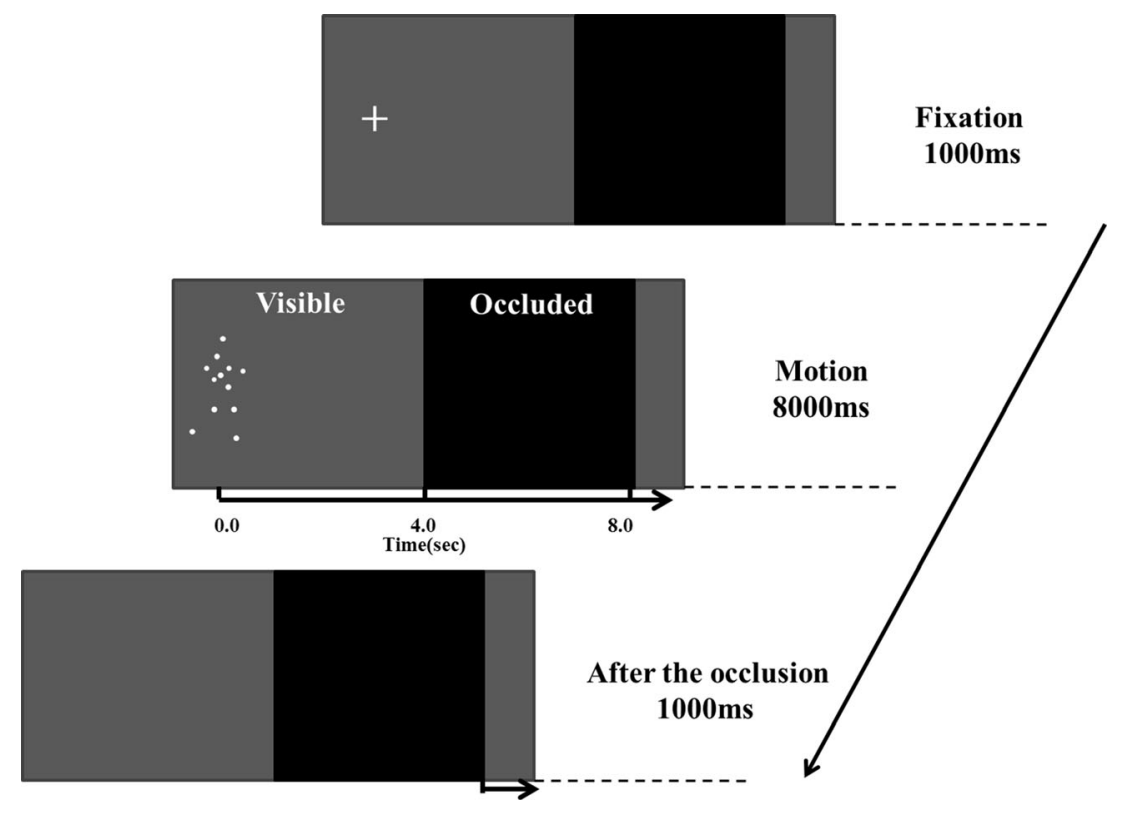

Fig. 2 Sequence of events in a trial

The eye movement data were scored offline. Blinks, drifts, and other artifacts were detected and eliminated from the oculomotor data ( $2.4 \%$ of responses in total). Corrective saccades were defined if their amplitude fell within the range of $0.8^{\circ}$ to $8^{\circ}$ (Haller, Fasler, Ohlendorf, Radue, \& Greenlee, 2008), and eye movements were defined as velocities faster than $40 \%$ s (Bennett \& Barnes, 2003). All data were extracted and stored separately. The cumulative saccade amplitude was about $5.3 \%$, on average. No significant differences in cumulative saccade amplitudes were found across conditions (see Table 1). Smooth pursuit was calculated by removing saccades and head movements from the eye gaze data. The smooth-pursuit velocity gain for each condition was calculated in terms of the peak velocities of the target and eye in a window of $100 \mathrm{~ms}$. Discrepancies were calculated between (1) the actual times that the first light marker disappeared at the left border of the bar or reappeared at the right side of the bar (known as the physical moving time) and (2) the times at which the participant indicated that the first light marker had reached the left side of the bar or reappeared at the right side of the bar (the recorded estimated times).

\section{Results}

\section{Behavior: predicted times}

Time discrepancies more than three standard deviations above or below the mean in each experimental condition were discarded as "outliers" (1.1\% of responses, in total). A Target Type (intact vs. scrambled) $\times$ Target Orientation (upright vs. inverted) $\times$ Target Visibility (visible vs. occluded) repeated measures analysis of variance (ANOVA) was conducted on the mean time discrepancies (see Fig. 3). The results showed a significant main effect of target type, $F(1,19)=$ 104.91, $p<.0001$, in which observers were more accurate to track the intact biological-motion target $(130 \mathrm{~ms})$ than the scrambled biological-motion target $(506 \mathrm{~ms})$. The main effect of target visibility was also significant, $F(1,19)=27.81, p<$ .0001 , in that time discrepancies were larger in memoryguided than in visually guided tracking trials (336 vs. $300 \mathrm{~ms}$ ). Although the main effect of target orientation was not significant, $F(1,19)=0.78, p>.1$, it nevertheless interacted with target visibility, $F(1,19)=38.22, p<.0001$. The interaction between target type and target visibility also reached significance, $F(1,19)=10.02, p<.01$. More

Table 1 Mean saccade amplitude (degrees) with standard errors (in parentheses) for each condition

\begin{tabular}{|c|c|c|c|c|}
\hline \multirow[t]{2}{*}{ Target Orientation } & \multicolumn{2}{|l|}{ Target Visible } & \multicolumn{2}{|c|}{ Target Occluded } \\
\hline & Intact Stimuli & Scrambled Stimuli & Intact Stimuli & Scrambled Stimuli \\
\hline Upright targets & $5.36(0.31)$ & $5.32(0.67)$ & $5.39(0.31)$ & $5.32(0.58)$ \\
\hline Inverted targets & $5.31(0.68)$ & $5.32(0.68)$ & $5.34(0.58)$ & $5.30(0.58)$ \\
\hline
\end{tabular}

The cumulative saccade amplitude was about $5.3 \mathrm{deg} / \mathrm{s}$ (average from 20 subjects). 


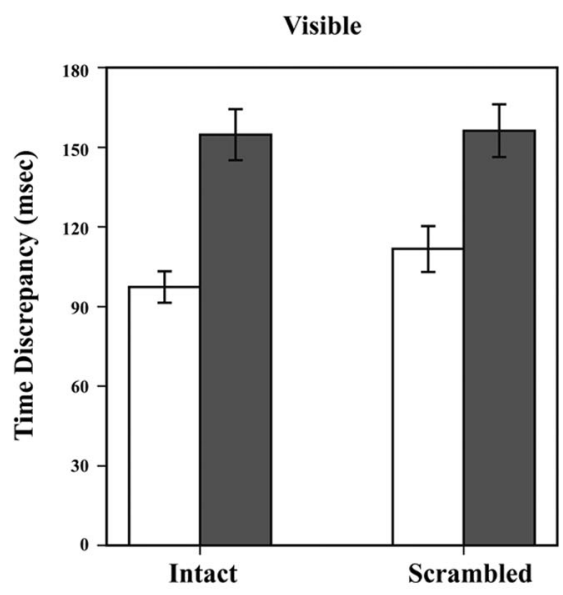

Target Type
Occluded

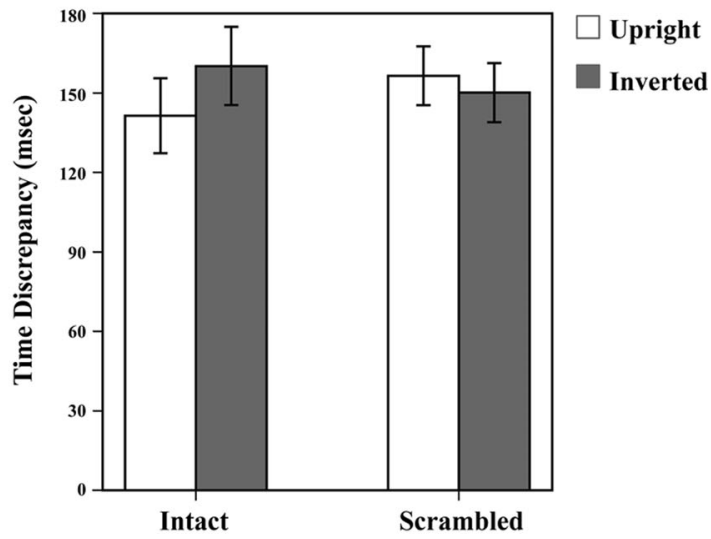

Target Type

Fig. 3 Mean time discrepancies (in milliseconds), with standard errors, as a function of the experimental conditions

importantly, the interaction between target type, target orientation, and target visibility was also significant, $F(2,14)=$ $9.38, p<.01$. These interactions suggested that the magnitude of the inversion effect of biological-motion processing was affected by both the target type and target visibility. No other main effects or interactions reached significance.

To examine these interactions, separate Target Type $\times$ Target Orientation ANOVAs were conducted for trials in which the target was visible and for trials the target was occluded. When the targets were visible, both the main effect of the target type and that of the target orientation were significant, $F(1,19)=4.74, p<.05 ; F(1,19)=73.58, p<.0001$, respectively. Time discrepancies were smaller for the intact $(126 \mathrm{~ms})$ than for the scrambled (134 ms) stimuli, and were smaller for the upright (104 ms) than for the inverted (155 ms) stimuli. More importantly, the interaction between the two factors reached marginal significance, $F(1,19)=4.12, p=$ .057 . Further analyses showed that the time discrepancies between upright and inverted trials differed not only in the intact biological-motion condition ( 97 vs. $154 \mathrm{~ms}$ ), $F(1,19)=65.67$, $p<.0001$, but also in scrambled biological-motion condition (111 vs. $156 \mathrm{~ms}), F(1,19)=49.41, p<.0001$.

When the targets were occluded, the main effect of target orientation was marginally significant, $F(1,19)=4.15, p=$ .056 , as was the target type and target orientation interaction, $F(1,19)=19.97, p<.0001$. Further analyses revealed that a significant inversion effect between the upright and inverted intact biological-motion conditions was evident in the time discrepancies (471 vs. $534 \mathrm{~ms}$ ), $F(1,19)=17.16, p<.001$. In contrast, there was no significant difference in time discrepancies between the upright and inverted scrambled biologicalmotion conditions (512 vs. $500 \mathrm{~ms}), F(1,19)=2.94, p>.1$, reflecting the fact that the inversion effect of scrambled biological-motion processing was affected by target visibility. Thus, during memory-guided tracking, the processing advantage for upright biological motion as compared with inverted biological motion was revealed not in the scrambled condition, but in the intact condition only.

\section{Eye movements: smooth-pursuit velocity gain}

Eye movements and behavioral data for each participant during the task were simultaneously recorded. The velocity gain was calculated as the ratio of the peak velocity of the recorded eye movement to the peak velocity of the moving target (see Fig. 4). A velocity gain of 1 means the target's velocity was tracked perfectly; if the gain is smaller than 1 , the eye velocity was slower than the moving target's velocity.

An ANOVA was conducted on the velocity gain, with Target Type (intact vs. scrambled), Target Orientation (upright vs. inverted), and Target Visibility (visible vs. occluded) as three within-subjects factors. The results showed a significant main effect of the target type, $F(1,19)=133.01, p<.0001$, and a significant main effect of the target orientation, $F(1,19)=$ $42.89, p<.0001$, with a higher gain on trials with intact stimuli than on trials with scrambled stimuli (.77 vs. .63), and higher gain on trials with upright stimuli than on trials with inverted stimuli (.73 vs. .68). The main effect of the target visibility was also significant, $F(1,19)=68.93, p<.0001$, suggesting that the overall gains were higher in target-visible than in targetoccluded trials (.73 vs. .68). Importantly, the Target Type $\times$ Target Visibility and the Target Type $\times$ Target Orientation $\times$ Target Visibility interactions were significant, $F(1,19)=6.15$, $p<.05$, and $F(1,19)=8.37, p<.01$, respectively. In other words, the gain differences between the two target orientations varied according to the target type and target visibility.

Separate ANOVAs, with Target Type and Target Orientation as two within-subjects factors, were performed for target-visible and target-occluded trials. For target-visible trials, the main effects of target type and target orientation were significant, $F(1,19)=26.86, p<.0001$, and $F(1,19)=51.23$, $p<.0001$. However, the two-way interaction between target 

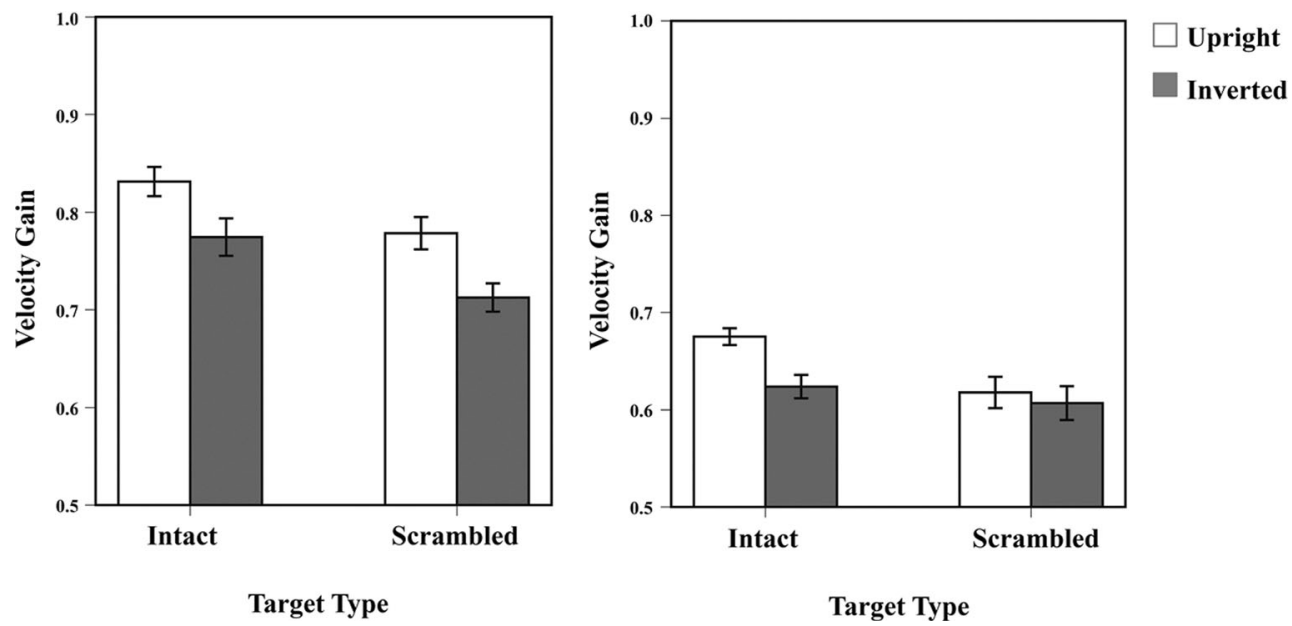

Fig. 4 Velocity gains for the visible phase (left panel) and the occluded phase (right panel)

type and target orientation did not reach significance, $F(1,19)$ $<1$, mainly because the average gains between the upright and inverted orientations revealed significant differences in the intact biological-motion condition (.83 vs. .77), $F(1,19)=$ $24.68, p<.0001$, as well as in the scrambled biologicalmotion condition (.78 vs. .71), $F(1,19)=25.67, p<.0001$.

For target-occluded trials, the main effects of target type and target orientation were significant, $F(1,19)=13.24, p<$ .005 , and $F(1,19)=15.49, p<.005$, respectively, as was the interaction between target type and target orientation, $F(1,19)$ $=11.56, p<.005$. As expected, it is clear from Fig. 4 that there was a significant difference between the upright and inverted trials in the intact biological-motion condition (.68 vs. .62), $F(1,19)=24.55, p<.0001$. In the scrambled biologicalmotion condition, however, we found no significant difference between the two target orientations (.62 vs. .61), $F(1$, 19) $=1.36, p>.1$. That is, the noticeable inversion effect disappeared during memory-guided smooth pursuit of scrambled biological motion.

\section{Discussion}

The human visual system is highly sensitive to biological motion. When point-light displays are presented upside down, tracking performance is strongly impaired. Moreover, the inversion effect of biological motion tracking was modulated by target type as well as by target visibility. Specifically, during the visually guided tracking, both observers' predicted times and predictive smooth pursuit were more accurate for upright biological motion (intact and scrambled) than for inverted biological motion. During memory-guided tracking, however, the processing advantage for upright as compared with inverted biological motion was not found in the scrambled condition, but in intact condition only. That is, the inversion effect disappeared when the scrambled biological motion was occluded.

The inversion effect is a widely demonstrated characteristic of biological motion processing (Bertenthal \& Pinto, 1994; Dittrich, 1993; Pavlova, 1989; Sumi, 1984). If point-light displays are inverted, observers' abilities to judge human action type (Dittrich, 1993; Shipley, 2003), walker direction (Bertenthal \& Pinto, 1994), and the agent's gender (Barclay, Cutting, \& Kozlowski, 1978) from them were also disrupted. The velocity gain was significantly lower for inverted than for upright biological motion (Coppe et al., 2010; Orban de Xivry, Coppe, Lefèvre, \& Missal, 2010). Indeed, we found a robust inversion effect for upright versus inverted biological motion during visually guided tracking. When tracking inverted intact biological motion, the time discrepancies between physical and predicted pursuit times increased, and the velocity gain decreased. Because the biological-motion stimuli moved from left to right with a constant velocity, leading to a predictable target trajectory, the smooth-pursuit system did not need to collaborate with the saccadic system in order to improve tracking continuity and accuracy (de Brouwer, Missal, Barnes, \& Lefèvre, 2002; de Brouwer, Yuksel, Blohm, Missal, \& Lefèvre, 2002; Erkelens, 2006; Laurutis, Daunys, \& Zemblys, 2010). Thus, we observed no differences in catch-up saccade amplitudes across conditions. Moreover, when asking observers to track the scrambled biologicalmotion stimuli, which obviously contained no global configuration but clearly kept the single-dot motion signals, the results were quite similar to those observed with intact biological motion (i.e., a noticeable inversion effect). Our results are consistent with those from previous studies using upright and inverted scrambled biological motion (Chang \& Troje, 2009; Troje \& Westhoff, 2006) and suggest that spatial location, which is processed in the "where" pathway, is sufficient for participants to successfully track biological motion at a high level. However, it is unknown whether memory-guided 
tracking of biological motion also relies solely on the updating of spatial location.

Previous studies have shown that the velocity gain was smaller during the occlusion phase than during the visible phase (Bennett \& Barnes, 2003; Bennett et al., 2007; Churchland, Chou, \& Lisberger, 2003; de Brouwer, Missal, \& Lefèvre, 2001; Ding et al., 2009; Mitrani \& Dimitrov, 1978), particularly when the stimuli conveyed obvious biological meaningfulness or biological significance (Coppe et al., 2010; Orban de Xivry et al., 2010). However, from the beginning to the end of the occlusion phase, the smoothpursuit velocity of observers did not reveal any significant difference between upright and inverted point-light walkers (Coppe et al., 2010). As was pointed out by Coppe and his colleagues (2010), the absence of a difference between the upright and inverted point-light walkers in velocity gains during memory-guided pursuit, and the stronger visually guided smooth pursuit reacceleration after reappearance of the upright point-light walker in comparison with the inverted point-light walker, suggests that during transient occlusion of biological motion, oculomotor prediction is primarily driven by the extraretinal inputs that contain the representation of the target spatial location (Bennett et al., 2010; Orban de Xivry et al., 2008).

According to the study by Coppe et al. (2010), attention to the upright point-light walker causes a set of nodes in LTM that represent target identity to be activated, and all history information about that target goes into an object file, which is capable of restoring continuity that has been briefly broken in the stream of sensory inputs. In contrast to the upright pointlight walker, the inverted point-light walker appeared for $800 \mathrm{~ms}$, which was not long enough to ensure sufficient processing time for the participants to build the identity representation and the identity-location binding of that global novelty target (the inverted point-light walker; Kahneman et al., 1992). However, the inversion of human figures as novelty stimuli to some extent engages more attentional resources, which in turn maintains tracking continuity during the transient absence of sensory inputs. Consequently, the processing advantages of biological meaningfulness or significance (arising from upright biological-motion signals) during memoryguided pursuit might have been offset by the attentional effects of the novelty stimulus (arising from inversion of human figures). To counterbalance a novelty bias for the inverted point-light walker, we extended the visible and occluded periods in the present experiment. In addition, we used both intact and scrambled biological motion, which were randomly presented in upright and inverted patterns. In the present data, both the behavioral and eye movement evidence indeed indicated that during memory-guided tracking, the processing advantage for upright as compared with inverted biological motion was not found in the scrambled condition, but only in the intact condition. In other words, the inversion effect disappeared only when the scrambled biological motion was occluded. According to Barnes and Collins (2008), when there is a complete loss of the visual feedback signal after target disappearance, smooth pursuit continues at a reduced gain and is maintained by extraretinal information processing. It has been recognized that the extraretinal input is a more complex arrangement reflecting velocity-coded information (Barnes \& Asselman, 1991; Bennett \& Barnes, 2004; Churchland et al., 2003), which is influenced by cognitive factors such as perception, expectation, and attention (Beutter \& Stone, 1998; Madelain \& Krauzlis, 2003; Pola \& Wyatt, 1997; Tanaka \& Lisberger, 2001). For the four biological-motion stimuli in the present study, velocity encoding is to some extent identical, whereas identity encoding differentiated by the global configuration is different. A number of previous studies have revealed that except for the upright intact biological motion, the other three biological-motion patterns contained no global configuration information but kept motion signals (Beintema, Georg, \& Lappe, 2006; Bertenthal \& Pinto, 1994; Hirai, Chang, Saunders, \& Troje, 2011; Lange \& Lappe, 2006; Wang, Zhang, He, \& Jiang, 2010). Therefore, the possible explanation for the disappearance of the inversion effect during memory-guided tracking scrambled biological motion is that the spatial location information is not sufficient to build and maintain the representation continuity about a scrambled point-light walker or its inverted version, and object identity may act as an important information source in visual tracking.

A recent study investigated the representation continuity for upright and inverted faces that also contain obvious biological significance. In the experiment by Ren et al. (2009), the participants were asked to track multiple faces, which were either upright images of the same individual, upright images of different individuals, or inverted images of different individuals. It was found that tracking was poorer for the upright different faces than for the inverted different faces (Ren et al., 2009). The findings contrast with our study, in which tracking performance was better if the intact point-light walkers were upright. A possible answer comes from considering the fundamental differences between human face and biologicalmotion processing. For example, complex facial features such as eyebrows and pigments are known to have a strong influence on face recognition (Sinha, Balas, Ostrovsky, \& Russell, 2006), whereas the local motion signals of feet are considered an essential component of biological-motion perception (Troje \& Westhoff, 2006). Facial features are often processed holistically, whereas biological-motion processing should be regarded as a multilevel phenomenon in which each level makes distinct contributions (Chang \& Troje, 2009; Saunders et al., 2009; see also Thornton, Pinto, \& Shiffrar, 1998). When the different faces are inverted, the abandonment of configural processing and face discrimination releases attentional resources for the tracking, hence the better tracking 
performance. However, this is not the case with the inverted intact point-light walker. The motion of biological entities consists of both local and global components. Even if the intact point-light walkers were inverted, local biological motion can also be processed independently of global configuration, which is available to some extent for the recovery of biological significance. Perhaps another answer is the difference between the experimental paradigms. In an experiment by Ren et al., participants were asked to track multiple faces, and the tracking performance for upright faces was strongly affected by the identity of the distractors. In our study, however, the task was to track single point-light walkers, and performance depended solely on the target characteristics per se.

Although the differences in the methodologies and experimental paradigms between Ren et al. (2009) and our study led to a reversal of the inversion effect, the results of two studies lend strong support to the object file theory (Kahneman et al., 1992; Oksama \& Hyönä, 2008), which involves the serial, nonautomatic, and effortful updating of identity-location binding (what-where binding). Location information comes from spatial indices, temporarily stored in VSTM, and identity from LTM representations (Botterill, Allen, \& McGeorge, 2010; Oksama \& Hyönä, 2008). With dynamic tracking of a point-light walker, the location information is always out of date and needs to be updated, because there will always be a spatial coordinate displacement between the actual target locations and the target location information stored in VSTM. However, the target identity is precisely what the target retains, even as its properties and the label or name we give it vary. The identity is said to be perceived when it activates a set of nodes in LTM that represent its parts, properties, and categories of membership (Kahneman, et al., 1992). Identity differentiated by semantic category (Horowitz et al., 2007; Yu, 2000) and global configuration (Botterill, Allen, \& McGeorge, 2010; De Caro, 1998; Ren et al., 2009) was encoded within a capacity limit and was accessible from higher-level cognitive processes (Oksama \& Hyönä, 2008). Dynamic identity-location binding is presumed to be held in working memory's episodic buffer, which makes recourse to continuous attention switching and helps to maintain the perceived continuity of objects as they move, change, or momentarily disappear (Kahneman et al., 1992).

In conclusion, in the present study we demonstrated a pronounced inversion effect during visually guided tracking of intact and scrambled biological motion. During memoryguided tracking, however, the inversion effect was found not in the scrambled condition, but in the intact condition only. This may be taken as evidence for the object file theory, which assumes that object identity acts as an important information source in visual tracking. When the biological motion is temporarily occluded and there is a complete absence of visual feedback signals, oculomotor prediction is executed to maintain the tracking continuity, which is established not only by the updating of target spatial location, but also by the retrieval of identity information stored in LTM.

Acknowledgments This work was supported in part by Beijing Social Science Foundation of China (No. 13ZHB008), and Open Research Fund of the State Key Laboratory of Cognitive Neuroscience and Learning.

\section{References}

Barclay, C. D., Cutting, J. E., \& Kozlowski, L. T. (1978). Temporal and spatial factors in gait perception that influence gender recognition. Perception \& Psychophysics, 23, 145-152.

Barnes, G. R., \& Asselman, P. T. (1991). The mechanism of prediction in human smooth pursuit eye movements. Journal of Physiology, 439, 439-461.

Barnes, G. R., Barnes, D. M., \& Chakraborti, S. R. (2000). Ocular pursuit responses to repeated, single-cycle sinusoids reveal behavior compatible with predictive pursuit. Journal of Neurophysiology, 84, 2340-2355.

Barnes, G. R., \& Collins, C. J. S. (2008). The influence of briefly presented randomized target motion on the extraretinal component of ocular pursuit. Neurophysiology, 99, 831-842.

Becker, W., \& Fuchs, A. F. (1985). Prediction in the oculomotor system: Smooth pursuit during transient disappearance of a visual target. Experimental Brain Research, 57, 562-575.

Beintema, J., Georg, K., \& Lappe, M. (2006). Perception of biological motion from limited-lifetime stimuli. Perception \& Psychophysics, 68, 613-624. doi:10.3758/BF03208763

Bennett, S. J., \& Barnes, G. R. (2003). Human ocular pursuit during the transient disappearance of a visual target. Journal of Neurophysiology, 90, 2504-2520.

Bennett, S. J., \& Barnes, G. R. (2004). Predictive smooth ocular pursuit during the transient disappearance of a visual target. Journal of Neurophysiology, 92, 578-590.

Bennett, S. J., Orban de Xivry, J. J., Barnes, G. R., \& Lefèvre, P. (2007). Target acceleration can be extracted and represented within the predictive drive to ocular pursuit. Journal of Neurophysiology, 98, 1405-1414. doi:10.1152/jn.00132.2007

Bennett, S. J., Orban de Xivry, J. J., Lefèvre, P., \& Barnes, G. R. (2010). Oculomotor prediction of accelerative target motion during occlusion: Long-term and short-term effects. Experimental Brain Research, 204, 493-504. doi:10.1007/s00221-010-2313-4

Bertenthal, B. I., \& Pinto, J. (1994). Global processing of biological motions. Psychological Science, 5, 221-225.

Beutter, B. R., \& Stone, L. S. (1998). Human motion perception and smooth eye movements show similar directional biases for elongated apertures. Vision Research, 38, 1273-1286.

Botterill, K., Allen, R., \& McGeorge, P. (2010). Multiple-object tracking: The binding of spatial location and featural identity. Experimental Psychology, 58, 196-200.

Bremner, J. G., Slater, A. M., Mason, U. C., Spring, J., \& Johnson, S. P. (2013). Trajectory perception and object continuity: Effects of shape and color change on 4-month-olds' perception of object identity. Development Psychology, 49, 1021-1026.

Carl, J. R., \& Gellman, R. S. (1987). Human smooth pursuit: Stimulusdependent responses. Journal of Neurophysiology, 57, 1446-1463.

Chang, D. H., \& Troje, N. F. (2009). Acceleration carries the local inversion effect in biological motion perception. Journal of Vision, 9(1), 19. doi:10.1167/9.1.19. 1-17. 
Churchland, A. K., Chou, I. H., \& Lisberger, S. G. (2003). Evidence for object permanence in the smooth-pursuit eye movements of monkeys. Journal of Neurophysiology, 90, 2205-2218.

Coppe, S., Orban de Xivry, J. J., Missal, M., \& Lefèvre, P. (2010). Biological motion influences the visuomotor transformation for smooth pursuit eye movements. Vision Research, 50, 2721-2728.

Cutting, J. (1978). A program to generate synthetic walkers as dynamic point-light displays. Behavior Research Methods \& Instrumentation, 10, 91-94.

de Brouwer, S., Missal, M., Barnes, G., \& Lefèvre, P. (2002a). Quantitative analysis of catch-up saccades during sustained pursuit. Journal of Neurophysiology, 87, 1772-1780.

de Brouwer, S., Missal, M., \& Lefèvre, P. (2001). Role of retinal slip in the prediction of target motion during smooth and saccadic pursuit. Journal of Neurophysiology, 86, 550-558.

de Brouwer, S., Yuksel, D., Blohm, G., Missal, M., \& Lefèvre, P. (2002b). What triggers catch-up saccades during visual tracking? Journal of Neurophysiology, 87, 1646-1650.

De Caro, S. A. (1998). On the perception of objects and their orientations. Spatial Vision, 11, 385-399.

Ding, J. H., Powell, D., \& Jiang, Y. (2009). Dissociable frontal controls during visible and memory-guided eye-tracking of moving targets. Human Brain Mapping, 30, 3541-3552.

Dittrich, W. H. (1993). Action categories and the perception of biological motion. Perception, 22, 15-22.

Erkelens, C. J. (2006). Coordination of smooth pursuit and saccades. Vision Research, 46, 163-170.

Farah, M. J., Tanaka, J. W., \& Drain, H. M. (1995). What causes the face inversion effect? Journal of Experimental Psychology: Human Perception and Performance, 21, 628-634. doi:10.1037/00961523.21.3.628

Haller, S., Fasler, D., Ohlendorf, S., Radue, E. W., \& Greenlee, M. W. (2008). Neural activation associated with corrective saccades during tasks with fixation, pursuit and saccades. Experimental Brain Research, 184, 83-94.

Hill, H., \& Pollick, F. E. (2000). Exaggerating temporal differences enhances recognition of individuals from point light displays. Psychological Science, 11, 223-228.

Hirai, M., Chang, D. H., Saunders, D. R., \& Troje, N. F. (2011). Body configuration modulates the usage of local cues to direction in biological-motion perception. Psychological Science, 22, 15431549.

Horowitz, T. S., Klieger, S. B., Fencsik, D. E., Yang, K. K., Alvarez, G. A., \& Wolfe, J. M. (2007). Tracking unique objects. Perception \& Psychophysics, 69, 172-184. doi:10.3758/BF03193740

Johansson, G. (1973). Visual perception of biological motion and a model for its analysis. Perception \& Psychophysics, 14, 201-211. doi:10. 3758/BF03212378

Kahneman, D., Treisman, A., \& Gibbs, B. J. (1992). The reviewing of object files: Object-specific integration of information. Cognitive Psychology, 24, 175-219. doi:10.1016/0010-0285(92)90007-O

Khoshafian, S. N., \& Copeland, G. P. (1986). Object identity. ACM SIGPLAN Notices, 21, 406-416.

Kozlowski, L. T., \& Cutting, J. E. (1977). Recognizing the sex of a walker from a dynamic point-light display. Perception \& Psychophysics, 21, 575-580. doi:10.3758/BF03198740

Lange, J., \& Lappe, M. (2006). A model of biological motion perception from configural form cues. Journal of Neuroscience, 26, 28942906.

Laurutis, V., Daunys, G., \& Zemblys, R. (2010). Quantitative analysis of catch-up saccades executed during two-dimensional smooth pursuit. Electronics and Electrical Engineering, 2, 83-86.

Leigh, R. J., \& Zee, D. S. (2006). The neurology of eye movements (4th ed.). Oxford, UK: Oxford University Press.

Lisberger, S. G., \& Fuchs, A. F. (1978). Role of primate flocculus during rapid behavioral modification of vestibuloocular reflex: I. Purkinje cell activity during visually guided horizontal smooth-pursuit eye movements and passive head rotation. Journal of Neurophysiology, 41, 733-763.

Madelain, L., \& Krauzlis, R. J. (2003). Effects of learning on smooth pursuit during transient disappearance of a visual target. Journal of Neurophysiology, 90, 972-982.

Mather, G., \& Murdoch, L. (1994). Gender discrimination in biological motion displays based on dynamic cues. Proceedings of the Royal Society B, 258, 273-279.

Mitrani, L., \& Dimitrov, G. (1978). Pursuit eye movements of a disappearing moving target. Vision Research, 18, 537-539.

Mrotek, L. A., \& Soechting, J. F. (2007). Predicting curvilinear target motion through an occlusion. Experimental Brain Research, 178, 99-114.

Muller, A. A., \& Aslin, R. N. (1978). Visual tracking as an index of the object concept. Infant Behavior and Development, 1, 309-319.

Norman, J. F., Payton, S. M., Long, J. R., \& Hawkes, L. M. (2004). Aging and the perception of biological motion. Psychology and Aging, 19, 219-225.

Oksama, L., \& Hyönä, J. (2008). Dynamic binding of identity and location information: A serial model of multiple identity tracking. Cognitive Psychology, 56, 237-283. doi:10.1016/j.cogpsych.2007. 03.001

Orban de Xivry, J. J., Bennett, S. J., Lefèvre, P., \& Barnes, G. R. (2006). Evidence for synergy between saccades and smooth pursuit during transient target disappearance. Journal of Neurophysiology, 95, 418-427.

Orban de Xivry, J. J., Coppe, S., Lefèvre, P., \& Missal, M. (2010). Biological motion drives perception and action. Journal of Vision, 10(2), 6. doi:10.1167/10.2.6. 1-11.

Orban de Xivry, J. J., Missal, M., \& Lefèvre, P. (2008). A dynamic representation of target motion drives predictive smooth pursuit during target blanking. Journal of Vision, 8(15), 6. doi:10.1167/8.15.6. 1-13.

Orban de Xivry, J. J., Missal, M., \& Lefèvre, P. (2009). Smooth pursuit performance during target blanking does not influence the triggering of predictive saccades. Journal of Vision, 9(11), 7. doi:10.1167/9.11. 7. 1-16.

Pavlova, M. (1989). The role of inversion in perception of biological motion pattern. Perception, 18, 510.

Pavlova, M., \& Sokolov, A. (2000). Orientation specificity in biological motion perception. Perception \& Psychophysics, 62, 889-899.

Pola, J., \& Wyatt, H. J. (1997). Offset dynamics of human smooth pursuit eye movements: Effects of target presence and subject attention. Vision Research, 37, 2579-2595.

Ren, D., Chen, W., Liu, C. H., \& Fu, X. (2009). Identity processing in multiple-face tracking. Journal of Vision, 9(5), 18. doi:10.1167/9.5. 18. 1-15.

Saunders, D. R., Suchan, J., \& Troje, N. F. (2009). Off on the wrong foot: Local features in biological motion. Perception, 38, 522-532. doi: $10.1068 / \mathrm{p} 6140$

Shipley, T. F. (2003). The effect of object and event orientation on perception of biological motion. Psychological Science, 14, 377-380.

Sinha, P., Balas, B., Ostrovsky, Y., \& Russell, R. (2006). Face recognition by humans: Nineteen results all computer vision researchers should know about. Proceedings of the IEEE, 94, 1948-1962. doi:10.1109/ JPROC.2006.884093

Sumi, S. (1984). Upside-down presentation of the Johansson moving light-spot pattern. Perception, 13, 283-286.

Tanaka, M., \& Lisberger, S. G. (2001). Regulation of the gain of visually guided smooth-pursuit eye movements by frontal cortex. Nature, 409, 191-194. 
Thornton, I. M., Pinto, J., \& Shiffrar, M. (1998). The visual perception of human locomotion. Cognitive Neuropsychology, 15, $535-552$.

Troje, N. F. (2002). Decomposing biological motion: A framework for analysis and synthesis of human gait patterns. Journal of Vision, 2(5), 2. doi:10.1167/2.5.2. 371-387.

Troje, N. F., \& Westhoff, C. (2006). The inversion effect in biological motion perception: Evidence for a "life detector"? Current Biology, 16, 821-824. doi:10.1016/j.cub.2006.03.022
Troje, N. F., Westhoff, C., \& Lavrov, M. (2005). Person identification from biological motion: Effects of structural and kinematic cues. Perception \& Psychophysics, 67, 667-675. doi:10.3758/BF03193523

Wang, L., Zhang, K., He, S., \& Jiang, Y. (2010). Searching for life motion signals: Visual search asymmetry in local but not global biological-motion processing. Psychological Science, $21,1083-1089$.

Yu, K. (2000). Can semantic knowledge influence motion correspondence? Perception, 29, 693-707. 